\title{
All eyes on rapid adaptive evolution
}

Many cave-dwelling animals adapt to their environment through the degeneration of eyesight or even complete eye loss. A new study reports that epigenetic repression of gene expression during development can underlie this trait regression.

While the eyeless morphs of Astyanax mexicanus (Mexican tetra) from the Pachón caves are morphologically indistinguishable from their surface-dwelling counterparts during initial stages of eye development, eye tissue degeneration initiates around 2 days after fertilization, leading to complete loss of eyes in adult cavefish. Genome analyses have failed to attribute this phenotypic variability to inactivating null mutations in genes essential for eye development, raising the possibility that eye gene expression is altered indirectly through cis-regulatory mutations that affect DNA methylation.

Expression of the DNA methyltransferase-encoding gene $d n m t 3 b b .1$ was increased 1.5 -fold in cavefish compared with surface fish as determined by reverse transcription quantitative PCR (RT-qPCR) and confirmed by RNA sequencing (RNA-seq). RNA-seq also showed reduced expression of a large number of eye development genes in cavefish compared with surface-living relatives. Zebrafish mutants lacking $d n m t 3 b b .1$ exhibited larger eye size and increased expression of several eye-relevant genes, including $c r x$, gnb3a and opn 1 lw 1 compared to wild-type control animals, suggesting that hypermethylation mediated by Dnmt3bb. 1 could underlie the repression of eye gene expression in cavefish.

RNA-seq and whole-genome bisulfite sequencing of genomic DNA identified 128 genes with markedly increased promoter methylation and decreased expression in cavefish versus surface fish embryonic eyes. Of these,

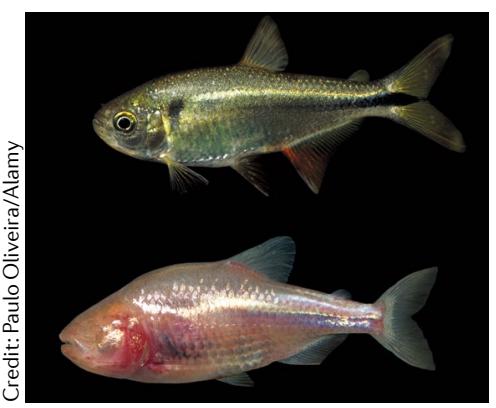

39 genes had been shown to be expressed in human eyes, with 19 genes having been linked previously to human eye disorders.

Looking at previously described zebrafish tet double mutants, which harbour genome-wide DNA hypermethylation, the authors found that increasing DNA methylation levels led to reduced eye gene expression and smaller eye size. This phenotype could be partially rescued by administration of 5-azacytidine, a well-known DNA methylation inhibitor, confirming the role of DNA methylation in teleost eye development.

\section{increasing DNA \\ methylation \\ levels led to reduced eye gene expression} Linda Koch

ORIGINAL ARTICLE Gore, A. V. et al.

An epigenetic mechanism for cavefish eye degeneration. Nat. Ecol. Evol. https://doi.org/ 10.1038/s41559-018-0569-4 (2018)

\section{What makes us human?}

Reference genomes for the great apes have suffered from limitations — including varying quality and completeness - that hamper the identification of genetic variation specific to humans. The publication in Science of new, improved great ape genome assemblies aims to address this issue.

Kronenberg et al. sequenced and assembled one chimpanzee and one orangutan genome, as well as two new human genomes, using high-coverage $(>65 \times)$ single-molecule real-time (SMRT) long-read sequencing. By scaffolding the chimpanzee and orangutan genomes without guidance from the human reference genome the authors avoid introducing a 'humanizing' bias, for example, during gene annotation. The resulting assemblies improve contiguity for the chimpanzee and orangutan genomes by 32 -fold and 533-fold, respectively.

De novo transcript models for chimpanzee, orangutan and gorilla genomes generated from full-length cDNA samples of induced pluripotent stem cells and short-read RNA sequencing data improved the mapping of human protein-coding transcripts to the chimpanzee and orangutan genomes, mainly as a result of closing existing gaps.

Five-way, genome-wide multiple sequence alignment showed that $83 \%$ of the ape genome was represented in the alignment. By mapping each assembly back to the human reference genome (GRCh38), using the two newly assembled human genomes as controls, the team could undertake comprehensive genome-wide analyses

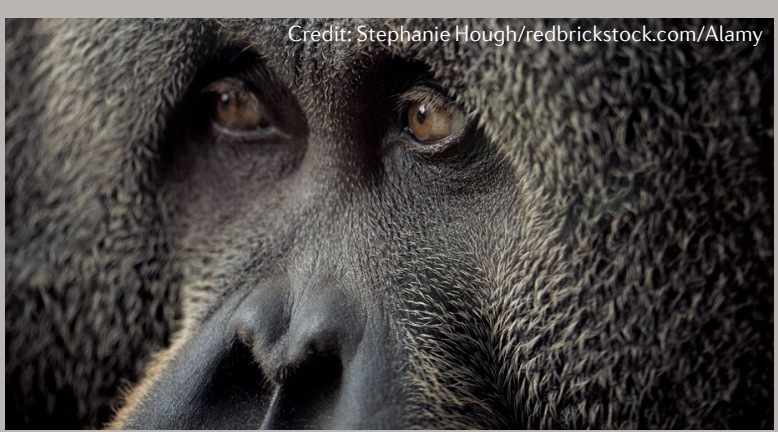

of structural variation (SV). They identified 614,186 deletions, insertions and inversions across the great apes, including 17,789 fixed human-specific SVs, 90 of which were predicted to disrupt genes, with an additional 643 potentially affecting regulatory regions.

Using single-cell gene expression data from human and chimpanzee cerebral organoid models and from primary human cortex, the authors compared SV locations with genes differentially expressed during brain development and found that $\sim 40 \%$ of genes downregulated in human radial glial neuroprogenitors were enriched for fixed SVs, mainly deletions or insertions. By contrast, genes associated with human-specific segmental duplications showed a pattern of upregulated expression. As the differential expression of genes during brain development may underlie the increase in brain volume from chimpanzees to humans over the course of evolution, functional analysis of the candidate genes highlighted by these associations may help identify the genetic differences that make us human. Linda Koch

ORIGINAL ARTICLE Kronenberg, Z. N. et al. High-resolution comparative analysis of great ape genomes. Science 360, eaar6343 (2018) 